\title{
Infrared ion spectroscopy in a modified quadrupole ion trap mass spectrometer at the FELIX free electron laser laboratory
}

Cite as: Rev. Sci. Instrum. 87, 103108 (2016); https://doi.org/10.1063/1.4964703

Submitted: 26 August 2016 . Accepted: 29 September 2016 . Published Online: 17 October 2016

Jonathan Martens (D, Giel Berden, Christoph R. Gebhardt, and Jos Oomens
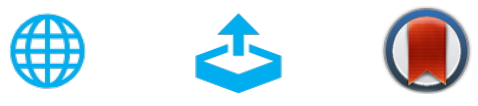

\section{ARTICLES YOU MAY BE INTERESTED IN}

Free electron laser-Fourier transform ion cyclotron resonance mass spectrometry facility for obtaining infrared multiphoton dissociation spectra of gaseous ions

Review of Scientific Instruments 76, 023103 (2005); https://doi.org/10.1063/1.1841953

Invited Article: Concepts and tools for the evaluation of measurement uncertainty

Review of Scientific Instruments 88, 011301 (2017); https://doi.org/10.1063/1.4974274

Hybrid quadrupole mass filter/quadrupole ion trap/time-of-flight-mass spectrometer for infrared multiple photon dissociation spectroscopy of mass-selected ions

Review of Scientific Instruments 82, 054101 (2011); https://doi.org/10.1063/1.3585982

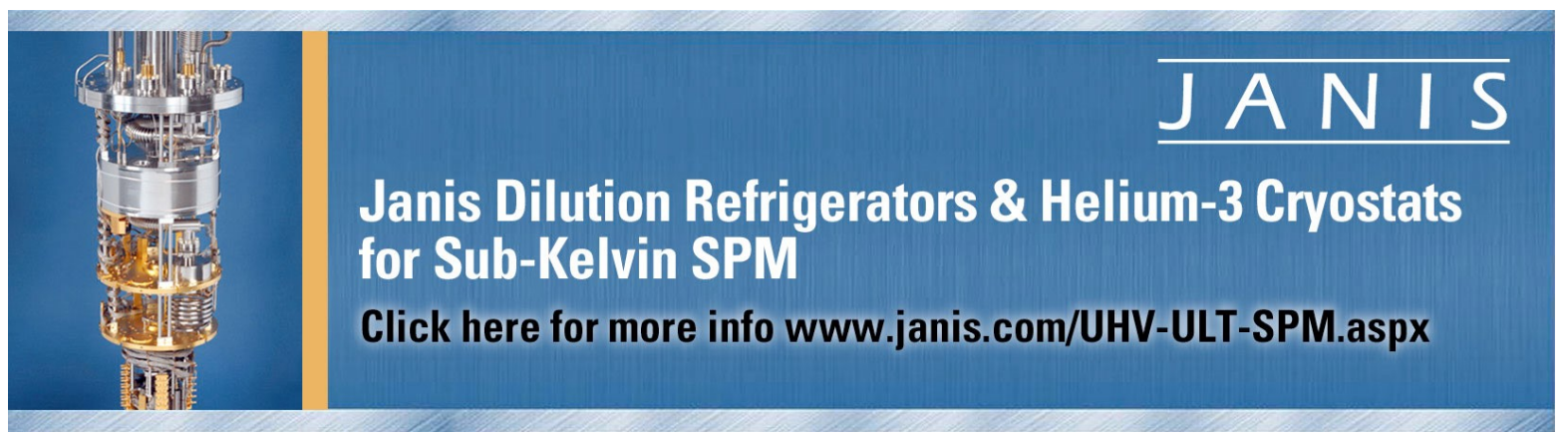




\title{
Infrared ion spectroscopy in a modified quadrupole ion trap mass spectrometer at the FELIX free electron laser laboratory
}

\author{
Jonathan Martens, ${ }^{1, a)}$ Giel Berden, ${ }^{1}$ Christoph R. Gebhardt, ${ }^{2, a)}$ and Jos Oomens ${ }^{1,3, a)}$ \\ ${ }^{1}$ Radboud University, Institute for Molecules and Materials, FELIX Laboratory, Toernooiveld 7c, \\ 6525ED Nijmegen, The Netherlands \\ ${ }^{2}$ Bruker Daltonik GmbH, Fahrenheitstrasse 4, D-28359 Bremen, Germany \\ ${ }^{3}$ Van't Hoff Institute for Molecular Sciences, University of Amsterdam, Science Park 908 , \\ 1098XH Amsterdam, The Netherlands
}

(Received 26 August 2016; accepted 29 September 2016; published online 17 October 2016)

\begin{abstract}
We report on modifications made to a Paul-type quadrupole ion trap mass spectrometer and discuss its application in infrared ion spectroscopy experiments. Main modifications involve optical access to the trapped ions and hardware and software coupling to a variety of infrared laser sources at the FELIX infrared free electron laser laboratory. In comparison to previously described infrared ion spectroscopy experiments at the FELIX laboratory, we find significant improvements in efficiency and sensitivity. Effects of the trapping conditions of the ions on the IR multiple photon dissociation spectra are explored. Enhanced photo-dissociation is found at lower pressures in the ion trap. Spectra obtained under reduced pressure conditions are found to more closely mimic those obtained in the high-vacuum conditions of an Fourier transform ion cyclotron resonance mass spectrometer. A gas-mixing system is described enabling the controlled addition of a secondary gas into helium buffer gas flowing into the trap and allows for ion/molecule reactions in the trap. The electron transfer dissociation (ETD) option of the mass spectrometer allows for IR structure characterization of ETD-generated peptide dissociation products. Published by AIP Publishing. [http://dx.doi.org/10.1063/1.4964703]
\end{abstract}

\section{INTRODUCTION}

Over the past decade, infrared ion spectroscopy has developed to become a powerful method for the characterization of molecular structure and conformation in ion chemistry and mass spectrometry (MS). This characterization is based on matching measured vibrational frequencies of ions trapped in a mass spectrometer with reference spectra, either calculated or measured for model compounds. ${ }^{1-8}$ Since infrared ion spectroscopy requires an intense and tunable infrared source, this technique has seen particularly widespread application at infrared free electron laser (FEL) facilities, ${ }^{1,9-11}$ but also tabletop sources such as optical parametric oscillators (OPOs) are in use in many labs.

Ion spectroscopy is an action technique, exploiting the mass spectrometer as a very sensitive detector of ion fragmentation resulting from resonant infrared absorption. This approach is used in place of measurement of the attenuation of the incident light beam because the number densities of mass/charge $(\mathrm{m} / \mathrm{z})$ selected ions are far too low to produce a measurable decrease of light intensity. The mass spectrometer thus serves as both the source of trapped ions and as the highly sensitive detector in such experiments. A variety of mass spectrometers has been used for these purposes, both home built and modified commercial instruments that provide optical access to trapped ions. Perhaps most notably, several Fourier transform ion cyclotron resonance (FT-ICR) mass

\footnotetext{
a)jonathan.martens@science.ru.nl; christoph.gebhardt@bruker.com; and j.oomens@science.ru.nl
}

spectrometers ${ }^{7,9,12-14}$ have been employed for this purpose. Both $3 \mathrm{D}^{15-21}$ and linear ${ }^{10,22-30}$ radio frequency (RF) traps have been used as platforms for infrared action spectroscopy experiments. Here, we report on hardware and software developments that allow for the coupling of an RF 3D ion trap mass spectrometer [Bruker amaZon speed ETD] to the FELIX free electron laser. This experiment offers significantly improved efficiency and sensitivity over previously existing infrared multiple photon dissociation (IRMPD) experiments at FELIX.

The instrument described here is one of the first reported examples of a mass spectrometer having electron transfer dissociation $^{31-33}$ (ETD) MS/MS capability coupled with infrared lasers, thus allowing for the structural characterization of ETD-generated peptide fragments by infrared ion spectroscopy. ${ }^{34}$ ETD takes advantage of the ability of radio frequency (RF) traps to simultaneously store the multiply charged analyte cation and a reagent anion, in this case the fluoranthene radical anion, ${ }^{35}$ so as to induce fragmentation of the analyte by electron transfer from the anion. However, despite the fact that much attention has been paid to ETD MS/MS in recent years, mechanistics of the process are still incompletely understood. ${ }^{36-38}$ The experimental apparatus described here provides the possibility of determining the structures of ETD MS/MS dissociation products, thus giving valuable information on the mechanisms by which they are formed.

The efficiency of IRMPD is highly dependent on the characteristics of the light source and the conditions that ions are trapped under. Relaxation of ions by collisional cooling directly competes with excitation by multiple-photon IR absorption, making the pressure of buffer gas in an RF-trap a critical parameter. Here we compare the effect of buffer 
gas pressure on IRMPD efficiency and the resulting spectra using both pulsed and continuous wave (cw) IR laser sources, reporting observations in line with previous reports. ${ }^{16,39,40}$ A detailed analysis of operating conditions and an in-depth description of the experimental apparatus are provided here.

\section{EXPERIMENTAL DESCRIPTION}

\section{Modified quadrupole ion trap mass spectrometer with optical access to trapped ions}

Figure 1 presents a schematic of the 3D quadrupole ion trap mass spectrometer discussed here. Ions are generated in an electrospray ionization (ESI) source consisting of the sprayer/nebulizer assembly and the spray chamber. Typically, solutions of $\sim 10^{-6} \mathrm{~mol} / \mathrm{l}$ are introduced at $120-180 \mu \mathrm{l} / \mathrm{hr}$ flow rates aided by a pressurized nebulizing gas $\left(\mathrm{N}_{2}\right)$. Additionally, a heated drying gas flows against the direction of the ions assisting desolvation and the removal of neutrals (typically, $180-220^{\circ} \mathrm{C}, 4-5 \mathrm{l} / \mathrm{min}$ ). The spray assembly is held at ground potential and ions are focused onto the entrance of a metal-coated glass capillary held $3-5 \mathrm{kV}$ below the sprayer and 200-500 V below the spray shield directly in front of the capillary entrance (for positive ions). When near the entrance to the capillary, ions are drawn into the capillary and transported further on due to the pressure gradient between the atmospheric pressure spray chamber and that of the first pumping stage at the exit of the capillary. The exit side of the capillary is also metal-coated and carries a potential of approximately 100-200 V. Ions exit the capillary directly into the first of two ion funnels. Their path from the capillary is off axis to the exit of the funnel to avoid contamination by neutrals at later stages in the mass spectrometer. The two funnels are separated by an additional DC plate which provides the possibility to accelerate the ions into the second funnel and obtain in-source collision induced dissociation (CID). After the funnels, ions are transferred using a multipole guide and finally pass through a gate and focusing lens assembly. Also, in this region, reagent radical anions for ETD experiments are extracted out of the chemical ionization (CI) source and combined with already trapped multiply charged cations.

During the accumulation time of typically $0.05-50 \mathrm{~ms}$, ions enter the trap through the first end-cap electrode where they are trapped by a radio-frequency field of 400-800 Vpp at $781 \mathrm{kHz}$ and cooled in $10^{-3} \mathrm{mbar}$ helium. Ions are finally scanned out of the trap onto a conversion dynode detector.

In ETD experiments, multiply charged cations are accumulated and mass isolated. Following this, a gate lens is switched along the main axis of the ion path to pass the ETD reagent anions from the CI source. Reagent anions are then briefly accumulated and undergo ion/ion reactions (here, typically for 50-300 ms) with the already trapped ESI-generated ions. A product or fragment from the ion/ion reaction can then be mass isolated in a subsequent MS/MS stage and subjected to IRMPD in a final MS/MS window. CID experiments proceed analogously; however, the ETD reaction stage is replaced by a short (typically $\sim 40 \mathrm{~ms}$ ) fragmentation stage where ions are collisionally activated to induce fragmentation.

Key modifications to the instrument providing optical access to the ion cloud involve the introduction of a modified ring electrode having two $3 \mathrm{~mm}$ holes centered in its top and bottom. As well, IR transparent windows (normally KRS-5) in the vacuum housing above the trap and two gold-coated mirrors below the trap were added so that a laser beam can pass through the trap, irradiating the ion population, and be guided back out of the instrument onto a sensor measuring the pulse energy, as illustrated in the inset of Figure 1. The introduction of the holed ring electrode increases the total area through which the helium buffer gas can escape the ion trap by a factor of 2.4. This naturally requires a substantially larger flow of helium into the trap to maintain the optimal

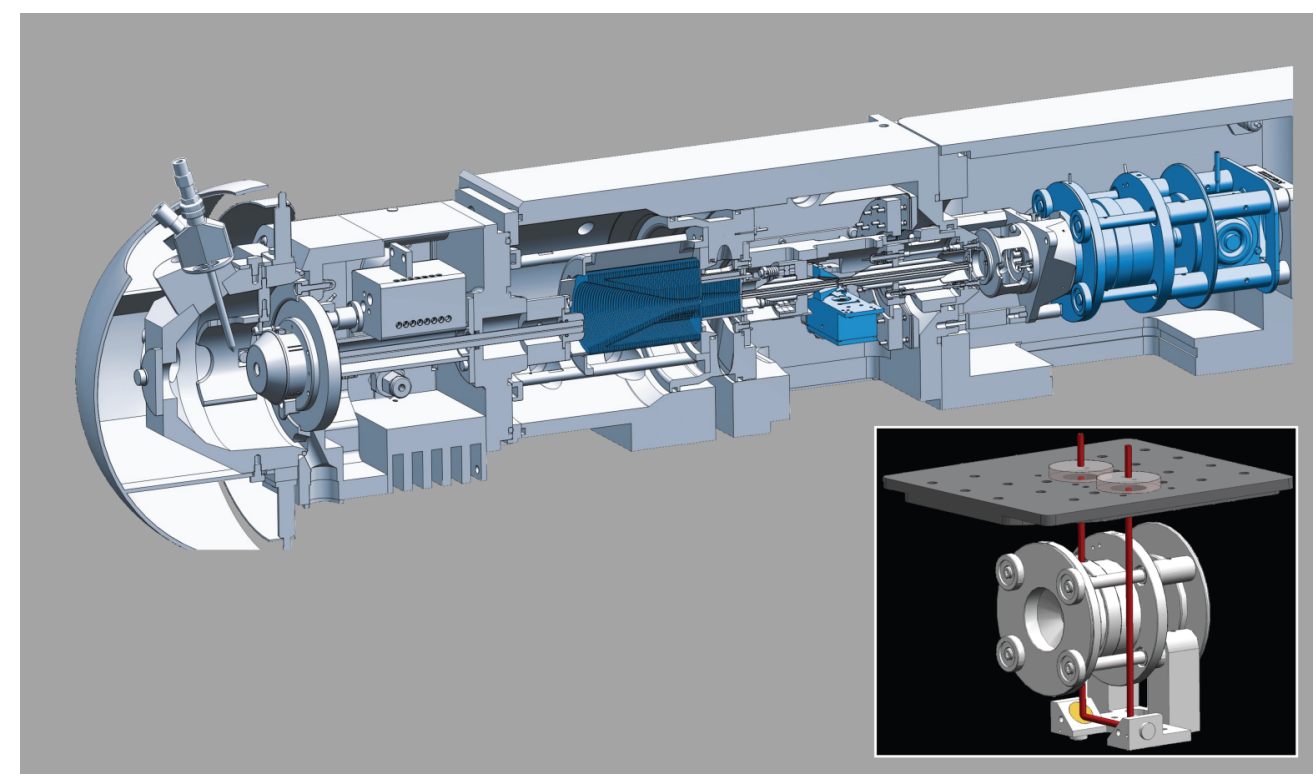

FIG. 1. The schematic of the quadrupole ion trap mass spectrometer and electrospray ionization source, with modifications for optical access to the trapped ions highlighted in the inset. The vacuum housing lid has been replaced with a small optical breadboard having two windows to pass the laser beam. The modified ring electrode has a $3 \mathrm{~mm}$ hole in its top-center and bottom-center. Two mirror mounts have been installed to guide the beam back out of the vacuum housing. 
equilibrium pressure for standard operation. We note that under these conditions, no reduction in the performance of the trap in terms of ion isolation, fragmentation, and resolution was observed to result from the introduction of the modified ring electrode. The primary gas controller used to regulate the helium flow into the trap, $\mathrm{GC}_{\mathrm{He}}$, consists of a closed loop controlled proportional valve that adjusts the pressure in front of a restriction to supply the required gas flows (low SCCM range). It is backed by helium at 5 bars and can be set between $0 \%$ and $100 \%$, where zero is off and 100 is the maximum flow giving high $10^{-3}$ mbar pressures in the trap. After installation of the modified ring electrode, the optimized value (for isolation, fragmentation, and mass resolution) was found to be $78 \%\left(\sim 10^{-3} \mathrm{mbar}\right)$.

\section{IRMPD experiment}

Figure 2 illustrates a typical MS sequence used during a standard IRMPD experiment on an electrosprayed ion. The black trace is the master trigger for the experiment, generated by the laser system and synchronized with the optical pulse; the MS sequence starts at one of these master trigger pulses. The green trace is the energy of the laser pulse monitored on a pyroelectric sensor after the beam exits from the trap. The pink trace is the signal monitored on an internal output of the driving circuit for the ion trap end caps and can be used to follow the MS sequence: (I) ion accumulation, (II) ion isolation, (III) an MS/MS window used for IRMPD, (IV) scanning ions out of the trap, and (V) waiting time before the mass spectrometer receives the next laser trigger and starts the next sequence. An additional fragmentation window would be added before the IRMPD window if the experiments were to involve CID or ETD generated ions. The blue trace shows a trigger generated by the mass spectrometer from the auxiliary interface during a user designated MS/MS (IRMPD) window, which is used to control a shutter in the optical beam path. As the total time of the MS sequence (accumulation, isolation, and possible MS/MS) before the IRMPD window varies, a delay generator is used to introduce a time difference between the laser trigger and the optical pulse to ensure that the laser pulses are centered in the IRMPD window and are not clipped at the beginning or end of the shutter opening/closing. Note that in this example, ions are irradiated with two laser pulses at a repetition rate of $10 \mathrm{~Hz}$ and the entire MS sequence takes $300 \mathrm{~ms}$, giving a three-fold averaged IR data point in approximately $1 \mathrm{~s}$ and a typical IRMPD spectrum over the full vibrational fingerprint region in $\sim 10-20 \mathrm{~min}$.

Synchronization is accomplished using software control through an XML scripting interface ${ }^{47}$ in the standard Bruker TrapControl program (unmodified) and an auxiliary interface on the mass spectrometer for hardware control. The instrument is set to start an averaged set of MS sequences only after receiving a software trigger while each of the individual MS sequences in the averaged set begins on a hardware trigger (external laser trigger, black trace in Figure 2) received on the instrument's auxiliary interface. The software trigger is sent to the trap by a home-written laser control program by saving the XML file in a directory monitored by TrapControl. This program sends the software trigger after each wavelength step of the infrared laser, also providing the current wavelength and pulse energy values to be saved with each averaged mass spectrum (see supplementary material for script details).

For the experiment described here, FELIX produces infrared radiation in the $600-1950 \mathrm{~cm}^{-1}$ region in the form of $5-10 \mu \mathrm{s}$ macropulses at $10 \mathrm{~Hz}$ which consist of a train of 6 ps long micropulses spaced by $1 \mathrm{~ns}$. The macropulses are approximately $40-100 \mathrm{~mJ} /$ pulse and have a bandwidth of $\sim 0.4 \%$ of the center frequency.

Apart from the free electron laser, two additional tunable infrared laser sources have been coupled to the ion trap instrument as well as a fixed wavelength $\mathrm{CO}_{2}$ laser. A pulsed Nd:YAG (InnoLas, Germany) pumped optical parametric oscillator/amplifier ${ }^{13}$ (OPO/A) (LaserVision, USA) has been implemented using an analogous synchronization scheme as presented above for FELIX. The OPO generates 5-ns pulses of $5-20 \mathrm{~mJ}$ at a $10 \mathrm{~Hz}$ repetition rate in the $2300-4000 \mathrm{~cm}^{-1}$ range with a bandwidth of approximately $3 \mathrm{~cm}^{-1}$. Additionally, a $50 \mathrm{~W}$ cw ytterbium fiber laser (IPG Photonics) pumped cwOPO (Lockheed Martin, USA) has been used which delivers up to $9 \mathrm{~W}$ in the $3100-4000 \mathrm{~cm}^{-1}$ range with a bandwidth

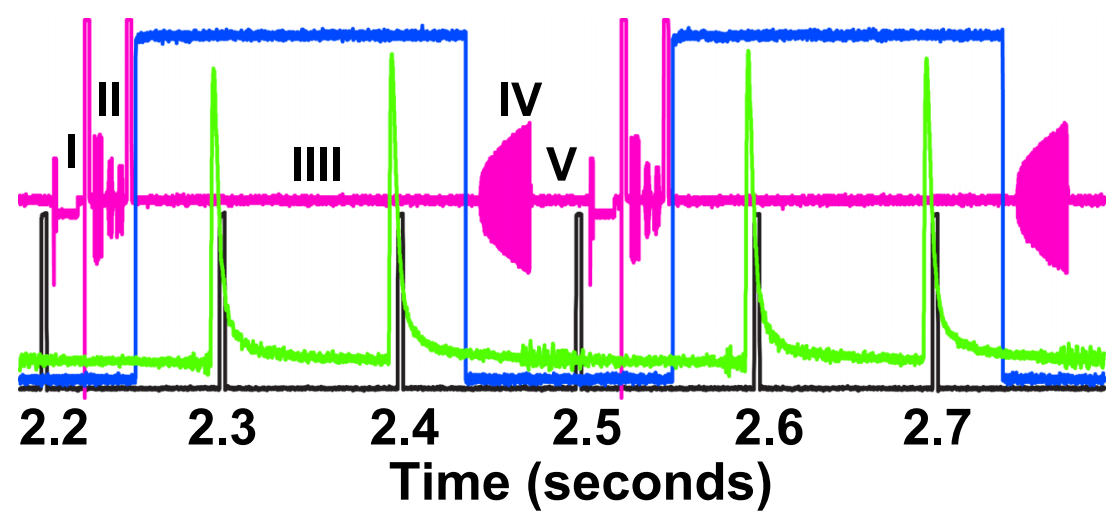

FIG. 2. Oscilloscope trace illustrating the synchronization of the MS-sequence with the laser pulse train. The pink trace is the voltage on the end caps and allows one to visualize each part of the MS-sequence. The blue trace is a trigger generated by the instrument for a selected component of the MS-sequence-here shown to control a shutter in the laser beam path, opening only during the time interval set as an IRMPD stage. The black trace is the master trigger (10 Hz) generated by the laser and the mass spectrometer starts each MS-sequence only after receiving this trigger. The green trace is the laser optical pulse measured on a detector after exiting the trap. Placement of the optical pulses within the MS-sequence is controlled by introducing a variable delay between the laser trigger synchronizing the instrument and the optical pulse of the laser. 
of $<6 \mathrm{~cm}^{-1}$. In this case, a fast galvo mirror at the exit of the cw-OPO is directly gated using the trigger from the mass spectrometer, which is kept in the "high" state for the duration of the appropriate MS/MS stage. Beam paths for the FEL and pulsed OPO enter the vacuum housing through the IR-transparent window directly above the trap. The cwOPO beam path is aligned counter-propagating and enters the vacuum housing through the mirror adjacent to the trap and is then directed into the trap from the bottom. This setup facilitates 2-color IR-IR experiments. Finally, a $35 \mathrm{~W}$ cw$\mathrm{CO}_{2}$ laser at a fixed wavelength of $10.6 \mu \mathrm{m}$ (Universal Laser Systems, USA) can be used for generating photodissociation products via IRMPD ${ }^{41}$ instead of CID or ETD. In this case, the laser is directly gated using the trigger from the mass spectrometer, which is kept in the "high" state for the duration of the appropriate MS/MS stage. The infrared spectrum of the product ion can then be obtained in a second MS window using the tunable FEL or OPO. Alternatively, the $\mathrm{CO}_{2}$ laser can be used to enhance the on-resonance dissociation yield while recording an infrared spectrum with the FEL or OPO (in case the FEL/OPO energy is not sufficient to dissociate the ions efficiently). In this scheme, the $\mathrm{CO}_{2}$ laser is synchronized to the FEL or OPO and the ions are irradiated for $0.1-20 \mathrm{~ms}$ with the $\mathrm{CO}_{2}$ laser directly after each FEL or OPO pulse. ${ }^{42,43}$

Resonant absorption of infrared radiation leads to an increase in the internal energy of a molecular system, which is followed by intramolecular vibrational redistribution (IVR), leading to randomization of the absorbed energy over all internal degrees of freedom. Typically, after the absorption of, on the order of 10 to a hundred photons, the system unimolecularly dissociates along the lowest energy pathway and produces frequency-dependent fragment intensities in the ion trap. Relating the parent and fragment ion intensities in the observed mass spectral data generates an infrared vibrational spectrum,

$$
\text { Yield }=\frac{\sum I_{\text {frags }}}{\sum I_{\text {all ions }}} .
$$

The yield at each IR point is obtained from 3 to 6 averaged mass spectra and is corrected for variations in laser power assuming a linear power dependence; the frequency is calibrated using a grating spectrometer (FELIX) or a wavemeter (OPOs). The various IR beams are focused using gold-plated spherical mirrors to give a beam waist of approximately $1.0-1.5 \mathrm{~mm}$ at the point of interaction with the ion cloud in the trap. The cw-OPO beam is not focused. The precise focus and alignment of the beams are routinely optimized for maximum precursor ion dissociation.

\section{Data analysis}

At each time step during acquisition, the time, IR wavelength, laser pulse energy, and the mass spectrum are recorded together. Analysis of the Bruker .d format data files is accomplished using the DataAnalysis program. Here the various extracted ion intensities over all steps, representing the spectral response of each $\mathrm{m} / \mathrm{z}$ channel, are generated, yields are calculated, and together with the laser wavelength and pulse energy values are directly written to a .csv file using an analysis script (see supplementary material for details of the script).

\section{Modifications for a secondary trap gas controller}

To create the possibility for ion/molecule and clustering reactions in the ion trap, a secondary trap gas controller, $\mathrm{GC}_{2}$, of similar type as $\mathrm{GC}_{\mathrm{He}}$ has been installed. $\mathrm{GC}_{\mathrm{He}}$ was removed from its direct connection to the trap on the main vacuum housing and installed on a Swagelok T-connector where one side was reconnected to the trap. On the remaining side of the T-connector, a high speed 3-way solenoid valve (Parker Series9) was installed with $\mathrm{GC}_{2}$ behind it. The third port of the valve is connected to a turbo molecular pump. A trigger pulse synchronizes the dosing of a reaction gas to the ion trap acquisition cycle. In this setup, $\mathrm{GC}_{2}$ (also carrying values from $0 \%$ to $100 \%$ ) is supplying a constant flow of reaction gas which is directed by the pulsed valve either into the waste or into the primary helium flow. Pulse widths on the $\geq 5 \mathrm{~ms}$ time scale are achievable, which is well below the values for direct flow changes via the controller. This can be used to add a reactant or clustering gas into the trap in a controlled manner. Figure 3 demonstrates hydrogen/deuterium (H/D) exchange of protonated phosphorylated tyrosine $(\mathrm{m} / \mathrm{z} 262)$ after the introduction of a relatively small amount of $\mathrm{ND}_{3}(5 \mathrm{~ms}$ pulse, $\mathrm{GC}_{2}=15 \%$ ), illustrating the potential for ion/molecule reactions in the trap.

\section{RESULTS AND DISCUSSION}

\section{Ion trapping conditions-Effects on measured IRMPD spectra}

As most ion trap mass spectrometers, the AmaZon platform employs a helium buffer gas pressure of typically $\sim 10^{-3}$ mbar to efficiently trap the ions and to collisionally quench the ion cloud to the center of the trap. However, the background pressure of $\mathrm{He}$ also has profound influence on the efficiency of IR multiple-photon dissociation; the competition between the IR excitation rate and the collisional de-excitation

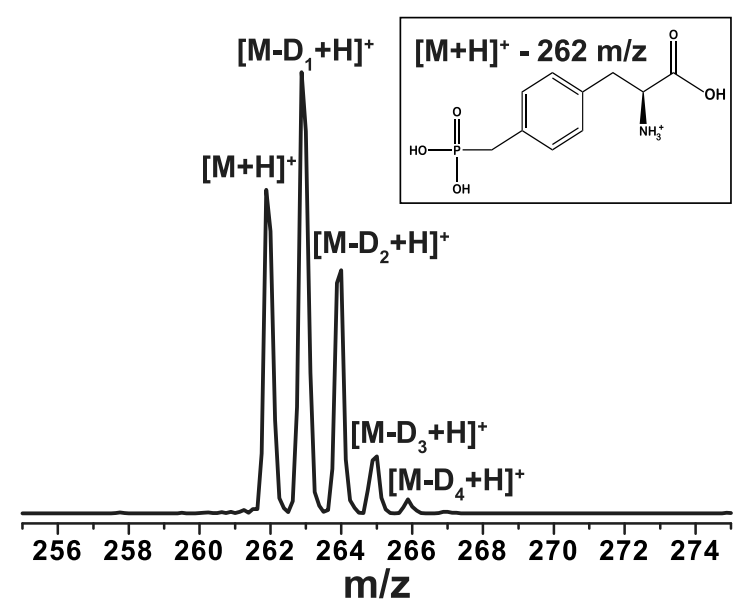

FIG. 3. Mass spectrum showing H/D exchange of protonated phosphotyrosine $\left(\mathrm{m} / \mathrm{z}\right.$ 262) using $\mathrm{ND}_{3}$ as a reagent. $\mathrm{ND}_{3}$ was introduced into the trap using a secondary gas controller, $\mathrm{GC}_{2}$, as described in the text. 
rate determines the final internal energy distribution that is reached after irradiation and from there the unimolecular dissociation rate. The fact that the instantaneous laser power is much higher from a pulsed-laser than from a cw-laser source strongly influences the competition between excitation and deexcitation rates, leading to substantial differences in IRMPD efficiencies when using either pulsed or cw-laser sources in traps with finite gas pressures. ${ }^{44}$

Figure 4(I) presents the total ion count observed as a function of the gas pressure (expressed as a percentile setting of the gas controller $\mathrm{GC}_{\mathrm{He}}$ ), clearly showing the rationale for using a He background pressure in these type of mass spectrometers. The curve in Figure 4(I) is in fact the sum of all ions during an IRMPD experiment presented in Figure 4(II), which shows the individual contributions of the precursor and several IR induced fragment ions. Longer ion accumulation times $(\sim 50 \mathrm{~ms})$ are used at the lowest $\mathrm{GC}_{\mathrm{He}}$ settings and shorter $(0.1 \mathrm{~ms})$ at higher $\mathrm{GC}_{\mathrm{He}}$ settings. Figure 4(II) clearly illustrates how heating by IR multiple-photon excitation competes with collisional cooling at increasing buffer gas pressures, to the extent where the system is hardly able to reach the dissociation threshold at the highest settings of $\mathrm{GC}_{\mathrm{He}},{ }^{39,45}$ normalized ion intensities of protonated tryptophan (m/z 205) and its two
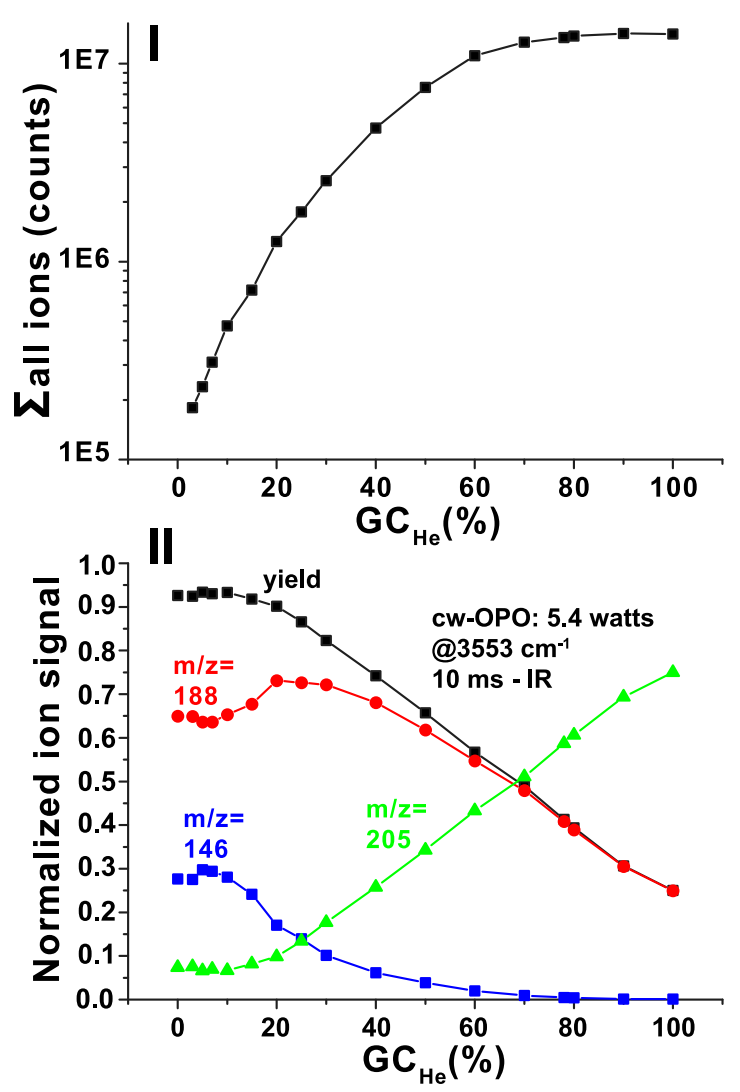

FIG. 4. Panel (I), the sum of all ion intensities (normalized for accumulation time) as a function of helium in the trap $\left(\mathrm{GC}_{\mathrm{He}}(\%)\right)$ for IRMPD photodissociation measurements of protonated tryptophan (m/z 205), panel (II). Note that while nearly two orders of magnitude are lost in signal intensity when helium is not used to cool ions entering the trap, more than sufficient signal remains for IRMPD spectroscopy measurements. Panel (II), the normalized ion signals and dissociation yield as a function of the pressure of helium in the trap for photodissociation measurements of protonated tryptophan $(\mathrm{m} / \mathrm{z}$ 205). dominant IRMPD fragment ions (m/z 146 is a sequential photo-fragment from $\mathrm{m} / \mathrm{z} 188$ ) using a cw-OPO source at $3550 \mathrm{~cm}^{-1}$ for $10 \mathrm{~ms}$ irradiation are shown as a function of buffer gas pressure. The IRMPD yield decreases from $\sim 0.93$ at low pressure conditions $\left(\sim 10^{-5}\right.$ mbar $)$, when $\mathrm{GC}_{\mathrm{He}}$ is disabled, to $\sim 0.25$ under slightly higher than typical operating pressures $\left(\sim 10^{-3}\right.$ mbar) when the $\mathrm{GC}_{\mathrm{He}}$ is operating at the maximum flow rate. Additionally, sequential photo-fragmentation of the $\mathrm{m} / \mathrm{z}$ 188 fragment ion to the $\mathrm{m} / \mathrm{z} 146$ fragment ion is completely suppressed at settings above $75 \%$ ( the optimal setting for normal MS operation).

Figure 5 presents the IRMPD spectra of protonated tryptophan measured in the AmaZon quadrupole ion trap using a pulsed-OPO, in panel (I) normal operating conditions $\left(\mathrm{GC}_{\mathrm{He}}-78 \%\right)$ with four IR laser pulses, in panel (II) low pressure conditions $\left(\mathrm{GC}_{\mathrm{He}}\right.$ - disabled, $\left.0 \%\right)$ with four laser pulses, in panel (III) reduced pressure conditions $\left(\mathrm{GC}_{\mathrm{He}}-\right.$ $20 \%$ ) with ten laser pulses, and finally in panel (IV) an analogous measurement using an FT-ICR MS under high vacuum conditions $\left(\sim 10^{-8}\right.$ mbar) and 45 laser pulses (chosen to obtain similar dissociation yields). ${ }^{46}$ Inlaid mass spectra show the relative intensities of the precursor and fragment ions at $3550 \mathrm{~cm}^{-1}$. Comparison of panels (I) and (II) shows the enhanced dissociation under low pressure conditions where the yield on all bands increases; however, this is accompanied by a substantial decrease in the signal to noise ratio due to the significantly decreased overall ion count. In panel (II), the lower pressure conditions presumably reduce the de-excitation rate and sequential photo-fragmentation of $\mathrm{m} / \mathrm{z} 188$ to $\mathrm{m} / \mathrm{z}$ 146 is observed extensively, as shown by the inlayed mass spectra. In comparison to the spectrum in panel (I), panel (II) is much closer to the spectrum measured in an FT-ICR MS (with background pressures in the $10^{-8}$ mbar range) shown in panel (IV). Reducing the pressure hence generates a more similar spectrum; however, intensities of the lower wavenumber bands (around $3100 \mathrm{~cm}^{-1}$ ) are still lower in relative intensity to those around 3500 and $3550 \mathrm{~cm}^{-1}$ in the spectra measured in the ion trap. In panel (III), the pressure is only somewhat lowered $\left(\mathrm{GC}_{\mathrm{He}}-20 \%\right)$ and 10 laser pulses are used. These settings are found to give some enhancement in IR dissociation, while maintaining a favorable signal to noise ratio and low background, while also giving a close match to the spectrum measured on the FT-ICR MS in panel (IV). ${ }^{45}$ The number of laser pulses and the setting of the $\mathrm{GC}_{\mathrm{He}}$, thus allows tuning of the balance between IR-excitation and collisional de-excitation.

Collisional quenching at higher pressures reducing the IRMPD efficiency can take drastic forms in species with higher dissociation thresholds. In Figure 6, the IRMPD spectrum of deprotonated para-coumaric acid (PCA) is displayed, focusing on the $\mathrm{OH}$ stretch band at $3590 \mathrm{~cm}^{-1}$. Spectra are recorded as a function of the setting $\mathrm{GC}_{\mathrm{He}}$ for $0 \%$ (disabled), 5\%, 20\%, and $78 \%$. In contrast to protonated tryptophan discussed above, which is readily dissociated even with the relatively low energy of the pulsed-OPO/A $(<20 \mathrm{~mJ})$, the dissociation threshold of deprotonated PCA is significantly higher and negligible fragmentation is observed under standard operating pressures in the trap (see curve marked "78" in Figure 6). In this case, reducing the $\mathrm{GC}_{\mathrm{He}}$ 

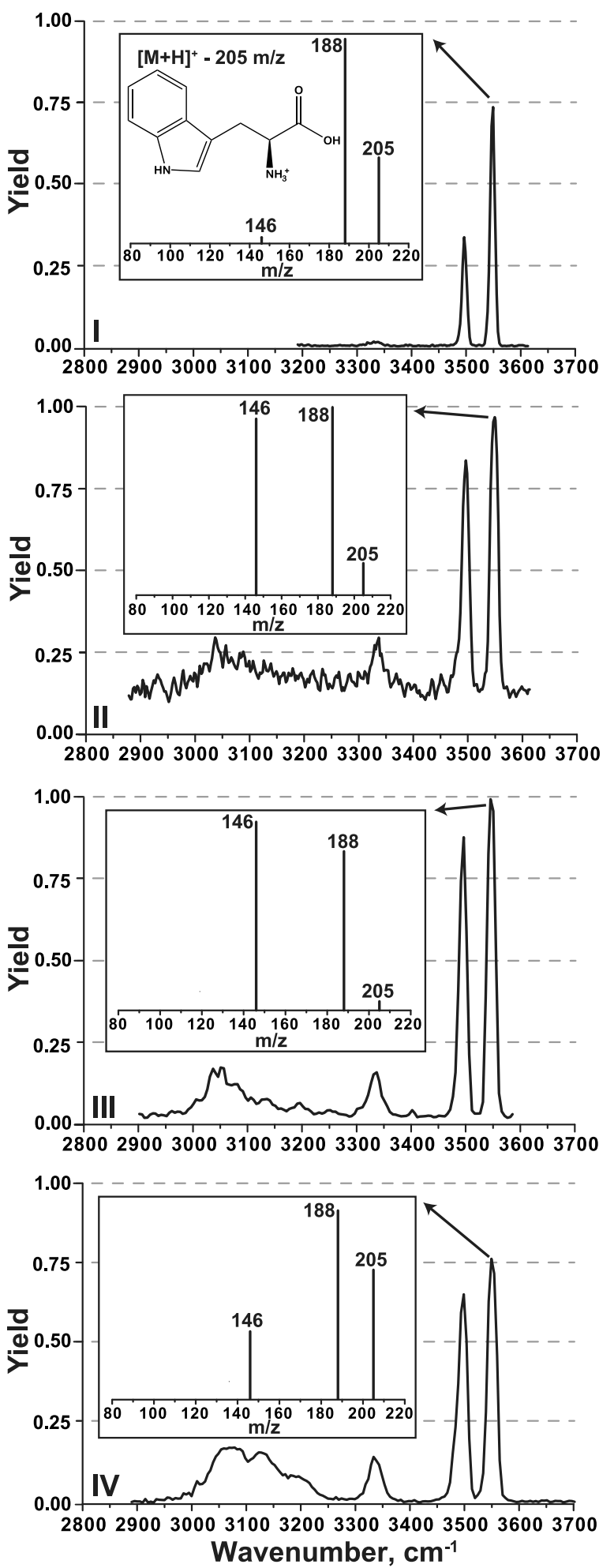

FIG. 5. IRMPD spectra of protonated tryptophan $(\mathrm{m} / \mathrm{z} 205)$ in the $3-\mu \mathrm{m}$ region measured using a pulsed-OPO. Comparison of spectra using different helium settings in the quadrupole ion trap and reference spectrum obtained on the FT-ICR MS in our laboratory, which operates at a lower pressure in the $10^{-8}$ mbar range, shows the balance between IR-excitation and collisional de-excitation. The insets show the mass spectra recorded at a frequency of $3553 \mathrm{~cm}^{-1}$.

operating parameter from $78 \%$ to $0 \%$ results in a nearly 10 times enhancement of the dissociation yield (along with a reduced overall ion count) and an approximate doubling of the signal/noise ratio.

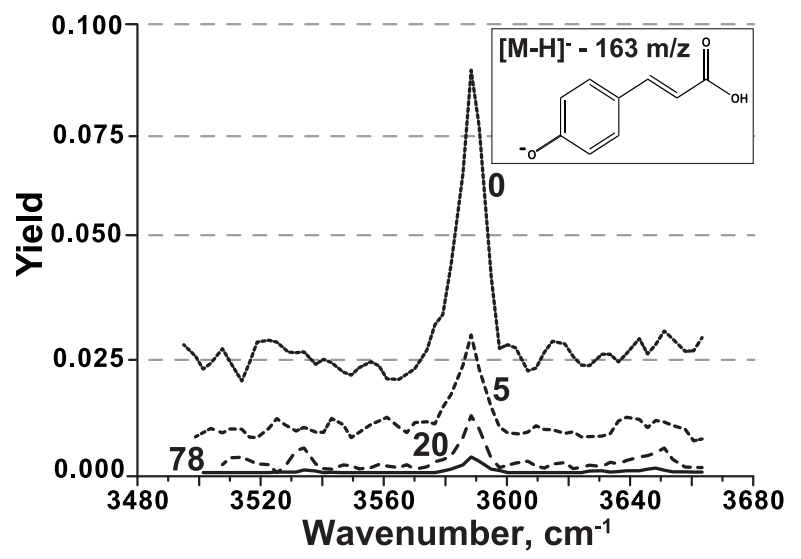

FIG. 6. IRMPD spectrum of the phenoxide isomer of deprotonated paracoumaric acid (PCA, m/z 163) using a pulsed-OPO and different pressures in the ion trap $\left(\mathrm{GC}_{\mathrm{He}}=0 \%, 5 \%, 20 \%\right.$, and $78 \%$, where the latter setting is the standard setting of the MS). The IRMPD spectra of PCA recorded in an FT-ICR MS (including the fingerprint region) can be found in the literature. ${ }^{42}$ The OPO pulse energy used for these experiments is approximately $15 \mathrm{~mJ} /$ pulse.

\section{Effect of trapping pressure and laser power using a continuous-wave laser source}

Figure 7 presents plots of normalized ion intensity and fragmentation yield as a function of irradiation time for different $\mathrm{GC}_{\mathrm{He}}$ settings (left- $0 \%$ to right- $-78 \%$ ) and different $\mathrm{cw}$-OPO laser powers (top- $5.4 \mathrm{~W}$, to bottom-1.35 W) for protonated tryptophan at $3553 \mathrm{~cm}^{-1}$. Comparing panels (I) and (III), we observe very similar behavior, an initial rise in the $\mathrm{m} / \mathrm{z} 188$ fragment followed by its decay and a rise in its photo-fragment at $\mathrm{m} / \mathrm{z} 146$. This leads eventually to complete conversion of all ion intensity to the $\mathrm{m} / \mathrm{z} 146$ product at $\sim 50 \mathrm{~ms}$ with $5.4 \mathrm{~W}$ (panel (I)) and at $\sim 200 \mathrm{~ms}$ with $1.35 \mathrm{~W}$ (panel (III)), corresponding directly to the factor of four decrease in laser power. Comparing panels (I) and (II), we again see similar behavior; however, under normal helium flow conditions (panel (II)), 50\% conversion of $\mathrm{m} / \mathrm{z}$ 188 to $\mathrm{m} / \mathrm{z} 146$ is achieved only after $100 \mathrm{~ms}$, whereas this is achieved under low-pressure conditions (panel (I)) in as little as $15 \mathrm{~ms}$, approximately seven times faster. Notice that complete depletion of the $\mathrm{m} / \mathrm{z} 205$ precursor ion is achieved in $\sim 20 \mathrm{~ms}$ in panel (I), indicating that all ions in the trap interact with the beam within this time period and once again demonstrates the excellent overlap of the ions with the beam, even without an elevated helium pressure in the trap. Comparison of panel (II) with (IV) demonstrates enhanced suppression of dissociation resulting from reduction of the laser power. In panel (II) with $5.4 \mathrm{~W}$ of IR power, the $\mathrm{m} / \mathrm{z}$ 205 precursor and $\mathrm{m} / \mathrm{z} 188$ primary fragment cross over at approximately $\sim 15 \mathrm{~ms}$, while for $1.35 \mathrm{~W}$, they cross over at $\sim 350 \mathrm{~ms}$, approximately 20 times longer. Under the conditions of higher pressure and reduced laser power (panel (IV)), formation of the secondary product is not observed, even at the longest irradiation times, indicative of a threshold behavior in the competition between IR heating and collisional cooling.

\section{ETD/IRMPD}

Figure 8 presents the infrared spectrum measured for the $\mathrm{z}_{2}{ }^{+}$radical sequence ion from the doubly protonated 

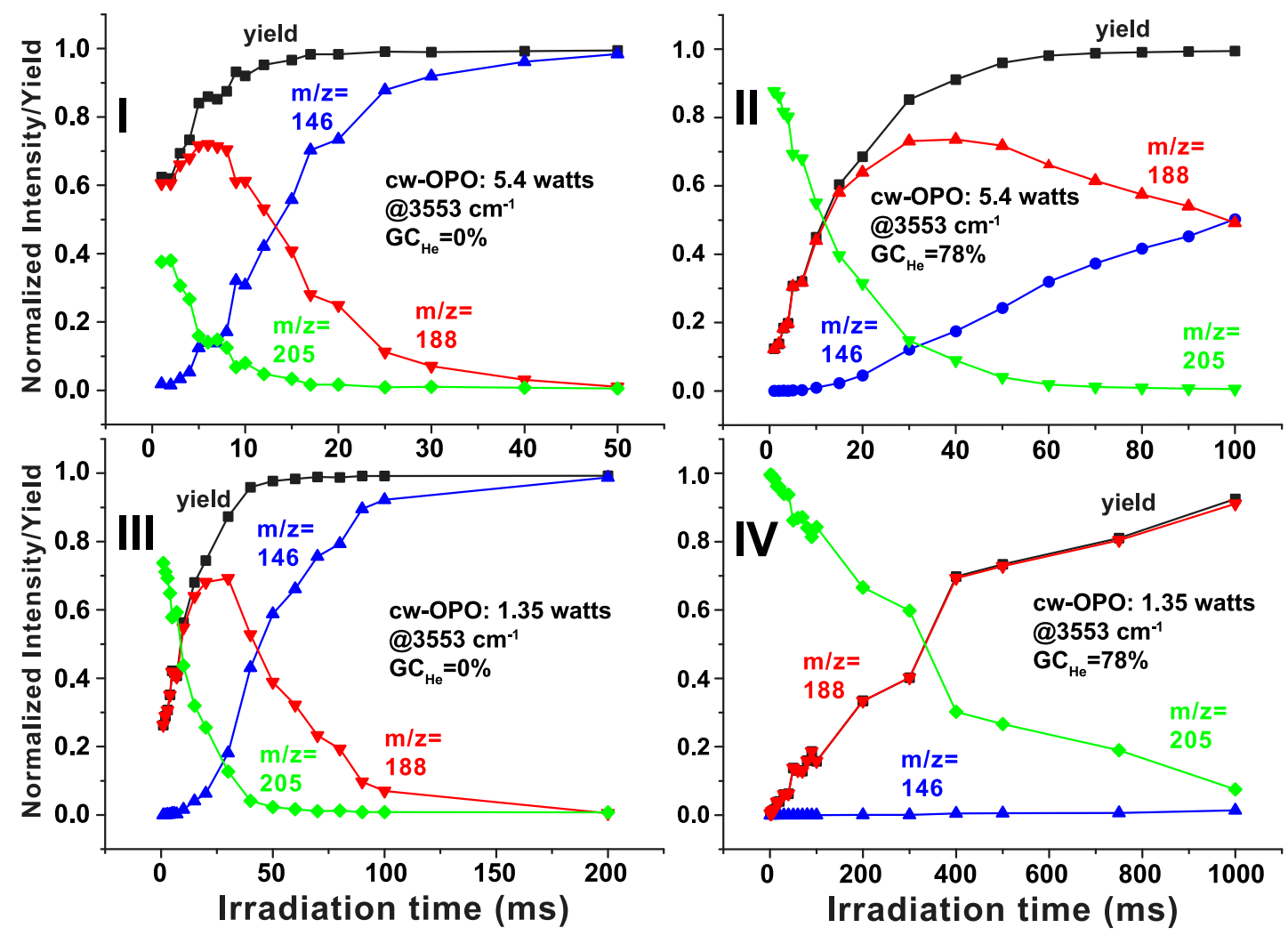

FIG. 7. Normalized ion intensities and dissociation yield of protonated tryptophan (m/z 205) under two different background pressures in the ion trap and two different laser powers of a cw-OPO source on resonance at $3553 \mathrm{~cm}^{-1}$.

AAHAR peptide by ETD, ${ }^{34}$ measured in the $1000-1900 \mathrm{~cm}^{-1}$ fingerprint region with FELIX and in the $2800-3700 \mathrm{~cm}^{-1}$ region using the pulsed-OPO described above. The fingerprint region spectrum was measured using two laser pulses and $5 \mathrm{~dB}$ attenuation $(10-20 \mathrm{~mJ} / \mathrm{pulse})$ of the FEL beam and the $3-\mu \mathrm{m}$ region was measured using 4 OPO laser pulses $(\sim 15 \mathrm{~mJ} /$ pulse between 3200 and $3700 \mathrm{~cm}^{-1}, \sim 10 \mathrm{~mJ} /$ pulse between 2800 and $3200 \mathrm{~cm}^{-1}$ ). Sharp bands are observed corresponding to the $\mathrm{O}-\mathrm{H}$ and $\mathrm{N}-\mathrm{H}$ stretches in the $3300-3600 \mathrm{~cm}^{-1}$ region as well as a broad feature of overlapping $\mathrm{C}-\mathrm{H}$ stretches just below $3000 \mathrm{~cm}^{-1}$. The band just below $1800 \mathrm{~cm}^{-1}$ is indicative of a non-hydrogen bonded carboxyl $\mathrm{C}=\mathrm{O}$. An analysis of the spectrum providing a structural characterization of this as well as other z-type sequence ions is published separately. ${ }^{34}$ While the ETD reaction undoubtedly results in a significant reduction (often approximately two orders of magnitude) in intensity going from precursor to fragment ions, IRMPD experiments on ETD-generated fragments are nevertheless possible with even moderate precursor ion intensities (above $\sim 10^{6}$ counts).

We note here that approximately complete depletion of the ion population is possible using a single FEL macropulse having an optical pulse duration of $\sim 5 \mu \mathrm{s}$, indicating nearly complete overlap of the ion cloud with the IR beam. Although, a single laser pulse often provides sufficient dissociation yields, in most cases, multiple 2-4 pulses (at attenuated power) are used to average out pulse to pulse fluctuations in laser power. It should also be noted that due to the favorable pulse structure of the FEL, the buffer gas in the trap can normally be maintained at typical operating values $\left(\mathrm{GC}_{\mathrm{He}}=78 \%\right)$ and is only reduced in rare cases with systems having very high

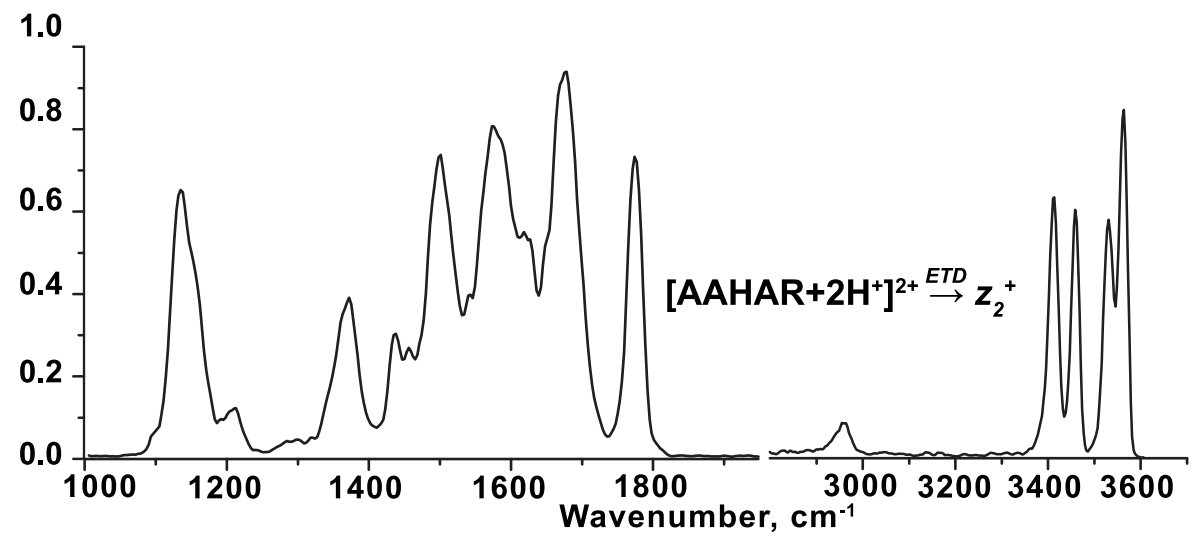

FIG. 8. IRMPD spectrum of the ETD-generated $\mathrm{z}_{2}{ }^{+}$fragment from the doubly protonated peptide $\left[\mathrm{AAHAR}+2 \mathrm{H}^{+}\right]^{2+}$, recorded with FELIX in the fingerprint region and with a pulsed-OPO in the $3-\mu$ m region. ${ }^{34}$ 
dissociation thresholds. Measurements in the $3-\mu \mathrm{m}$ range using the pulsed-OPO typically use 2-20 laser pulses.

\section{CONCLUSIONS}

We describe the implementation and optimization of a new IRMPD spectroscopy experiment based on a modified quadrupole ion trap mass spectrometer at the FELIX Laboratory. A favorable interaction between the IR beam and ion cloud is found for the purpose of recording IR ion spectra. Typically, optimal IRMPD yields are obtained using a small number (1-4) of FEL pulses, or 2-20 pulses of a LaserVision OPO; using a cw-OPO, irradiation for 5-1000 ms induces sufficient fragmentation for ion spectroscopy purposes. Reducing the buffer gas pressure in the trap is found to provide dramatically enhanced dissociation yields, especially in the cases when an ion has a relatively high dissociation threshold, with a continuous-wave IR source, or under lower power conditions. Furthermore, with the MS/MS capabilities of the ion trap MS, we show that it is among other things, now possible to record spectra for product ions generated by H/D exchange or by ETD of multiply protonated peptides, which in combination with quantum-chemical calculations will form a strong asset in their structural characterization.

\section{SUPPLEMENTARY MATERIAL}

Examples of scripts used for managing the interfacing and synchronization of the mass spectrometer with a laser system as well as for data analysis are available in the online supplementary material.

\section{ACKNOWLEDGMENTS}

The authors gratefully acknowledge the FELIX staff, particularly Dr. B. Redlich, Dr. A. F. G. van der Meer, R. van Buuren, A. J. H. Tielemans, A. P. van Vliet, and W. D. H. Stumpel for technical support. Financial support for this project was provided by NWO Chemical Sciences under VICI Project No. 724.011.002. The authors also thank NWO Physical Sciences (EW). This work is part of the research program of FOM, which is financially supported by NWO.

${ }^{1}$ J. Lemaire, P. Boissel, M. Heninger, G. Mauclaire, G. Bellec, H. Mestdagh, A. Simon, S. L. Caer, J. M. Ortega, F. Glotin, and P. Maitre, Phys. Rev. Lett. 89(27), 273002 (2002).

${ }^{2}$ J. Oomens, B. G. Sartakov, G. Meijer, and G. von Helden, Int. J. Mass Spectrom. 254(1-2), 1-19 (2006).

${ }^{3}$ N. C. Polfer and J. Oomens, Mass Spectrom. Rev. 28(3), 468-494 (2009).

${ }^{4}$ N. C. Polfer, Chem. Soc. Rev. 40(5), 2211-2221 (2011).

${ }^{5}$ N. C. Polfer and J. Oomens, Phys. Chem. Chem. Phys. 9(29), 3804-3817 (2007).

${ }^{6}$ J. R. Eyler, Mass Spectrom. Rev. 28(3), 448-467 (2009).

${ }^{7}$ T. D. Fridgen, Mass Spectrom. Rev. 28(4), 586-607 (2009).

${ }^{8}$ L. MacAleese and P. Maître, Mass Spectrom. Rev. 26(4), 583-605 (2007).

${ }^{9}$ J. J. Valle, J. R. Eyler, J. Oomens, D. T. Moore, A. F. G. van der Meer, G. von Helden, G. Meijer, C. L. Hendrickson, A. G. Marshall, and G. T. Blakney, Rev. Sci. Instrum. 76(2), 023103 (2005).

${ }^{10}$ S. Warnke, J. Seo, J. Boschmans, F. Sobott, J. H. Scrivens, C. Bleiholder, M. T. Bowers, S. Gewinner, W. Schöllkopf, K. Pagel, and G. von Helden, J. Am. Chem. Soc. 137(12), 4236-4242 (2015).

${ }^{11}$ Y. Miyamoto, T. Majima, S. Arai, K. Katsumata, H. Akagi, A. Maeda, H. Hata, K. Kuramochi, Y. Kato, and K. Tsukiyama, Nucl. Instrum. Methods Phys. Res., Sect. B 269(2), 180-184 (2011).
${ }^{12}$ G. Mauclaire, J. Lemaire, P. Boissel, G. Bellec, and M. Heninger, Eur. J. Mass Spectrom. 10(2), 155-162 (2004).

${ }^{13}$ J. M. Bakker, T. Besson, J. Lemaire, D. Scuderi, and P. Maître, J. Phys. Chem. A 111(51), 13415-13424 (2007).

${ }^{14}$ M. F. Bush, J. T. O’Brien, J. S. Prell, R. J. Saykally, and E. R. Williams, J. Am. Chem. Soc. 129(6), 1612-1622 (2007).

${ }^{15}$ L. M. Aleese, A. Simon, T. B. McMahon, J.-M. Ortega, D. Scuderi, J. Lemaire, and P. Maître, Int. J. Mass Spectrom. 249-250, 14-20 (2006).

${ }^{16}$ K. Gulyuz, C. N. Stedwell, D. Wang, and N. C. Polfer, Rev. Sci. Instrum. 82(5), 054101 (2011).

${ }^{17}$ J. Oomens, A. J. A. v. Roij, G. Meijer, and G. v. Helden, Astrophys. J. 542(1), 404 (2000).

${ }^{18}$ R. K. Sinha, P. Maitre, S. Piccirillo, B. Chiavarino, M. E. Crestoni, and S. Fornarini, Phys. Chem. Chem. Phys. 12(33), 9794-9800 (2010).

${ }^{19}$ P. Maitre, J. Lemaire, and D. Scuderi, Phys. Scr. 78(5), 058111 (2008).

${ }^{20}$ Y. Nosenko, F. Menges, C. Riehn, and G. Niedner-Schatteburg, Phys. Chem. Chem. Phys. 15(21), 8171-8178 (2013).

${ }^{21}$ B. Schindler, J. Joshi, A.-R. Allouche, D. Simon, S. Chambert, V. Brites, M.-P. Gaigeot, and I. Compagnon, Phys. Chem. Chem. Phys. 16(40), 22131-22138 (2014).

${ }^{22}$ K. R. Asmis, M. Brummer, C. Kaposta, G. Santambrogio, G. von Helden, G. Meijer, K. Rademann, and L. Woste, Phys. Chem. Chem. Phys. 4(7), 1101-1104 (2002).

${ }^{23}$ M. Z. Kamrath, R. A. Relph, T. L. Guasco, C. M. Leavitt, and M. A. Johnson, Int. J. Mass Spectrom. 300(2-3), 91-98 (2011).

${ }^{24}$ A. B. Wolk, C. M. Leavitt, E. Garand, and M. A. Johnson, Acc. Chem. Res. 47(1), 202-210 (2014).

${ }^{25}$ O. Asvany, F. Bielau, D. Moratschke, J. Krause, and S. Schlemmer, Rev. Sci. Instrum. 81(7), 076102 (2010).

${ }^{26}$ O. Asvany, S. Brünken, L. Kluge, and S. Schlemmer, Appl. Phys. B 114(1), 203-211 (2014).

${ }^{27}$ J. G. Redwine, Z. A. Davis, N. L. Burke, R. A. Oglesbee, S. A. McLuckey, and T. S. Zwier, Int. J. Mass Spectrom. 348, 9-14 (2013).

${ }^{28}$ O. V. Boyarkin, S. R. Mercier, A. Kamariotis, and T. R. Rizzo, J. Am. Chem. Soc. 128(9), 2816-2817 (2006).

${ }^{29}$ G. Papadopoulos, A. Svendsen, O. V. Boyarkin, and T. R. Rizzo, Faraday Discuss. 150, 243-255 (2011).

${ }^{30}$ J. Jašík, J. Žabka, J. Roithová, and D. Gerlich, Int. J. Mass Spectrom. 354-355, 204-210 (2013).

${ }^{31}$ J. E. P. Syka, J. J. Coon, M. J. Schroeder, J. Shabanowitz, and D. F. Hunt, Proc. Natl. Acad. Sci. U. S. A. 101(26), 9528-9533 (2004).

${ }^{32}$ J. J. Coon, B. Ueberheide, J. E. P. Syka, D. D. Dryhurst, J. Ausio, J. Shabanowitz, and D. F. Hunt, Proc. Natl. Acad. Sci. U. S. A. 102(27), 9463-9468 (2005).

${ }^{33}$ S. A. McLuckey and T.-Y. Huang, Anal. Chem. 81(21), 8669-8676 (2009).

${ }^{34}$ J. Martens, J. Grzetic, G. Berden, and J. Oomens, Nat. Commun. 7, 11754 (2016).

${ }^{35}$ J. Martens, G. Berden, and J. Oomens, Anal. Chem. 88(12), 6126-6129 (2016).

${ }^{36}$ F. Tureček and R. R. Julian, Chem. Rev. 113(8), 6691-6733 (2013).

${ }^{37}$ J. Simons, Chem. Phys. Lett. 484(4-6), 81-95 (2010).

${ }^{38}$ I. Anusiewicz, P. Skurski, and J. Simons, J. Phys. Chem. B 118(28), 7892-7901 (2014).

${ }^{39}$ P. M. Remes and G. L. Glish, Int. J. Mass Spectrom. 265(2-3), 176-181 (2007).

${ }^{40}$ G. A. Newsome and G. L. Glish, J. Am. Soc. Mass Spectrom. 20(6), 1127-1131 (2009).

${ }^{41}$ J. K. Martens, J. Grzetic, G. Berden, and J. Oomens, Int. J. Mass Spectrom. 377, 179-187 (2015).

${ }^{42}$ M. Almasian, J. Grzetic, J. van Maurik, J. D. Steill, G. Berden, S. Ingemann, W. J. Buma, and J. Oomens, J. Phys. Chem. Lett. 3(16), 2259-2263 (2012).

${ }^{43}$ R. K. Sinha, U. Erlekam, B. J. Bythell, B. Paizs, and P. Maître, J. Am. Soc. Mass Spectrom. 22(9), 1645-1650 (2011).

${ }^{44}$ A. M. Rijs and J. Oomens, in Gas-Phase IR Spectroscopy and Structure of Biological Molecules, edited by M. A. Rijs and J. Oomens (Springer International Publishing, Cham, 2015), pp. 1-42.

${ }^{45}$ O. Hernandez, B. Paizs, and P. Maître, Int. J. Mass Spectrom. 377, 172-178 (2015).

${ }^{46}$ W. K. Mino, K. Gulyuz, D. Wang, C. N. Stedwell, and N. C. Polfer, J. Phys. Chem. Lett. 2(4), 299-304 (2011).

${ }^{47}$ Enabling the XML interface requires operating TrapControl in "service" mode, for details contact a Bruker representative. 